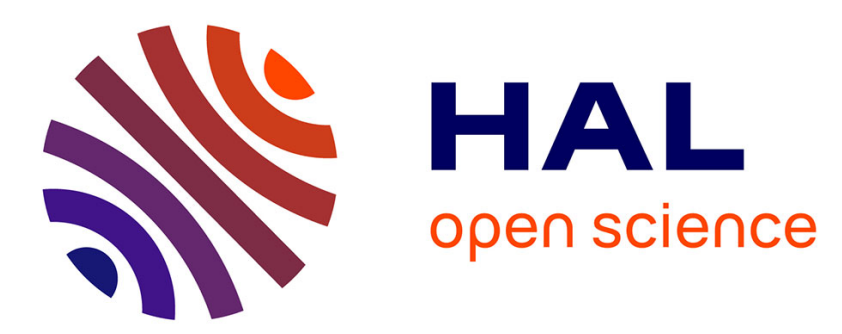

\title{
Assessment of Aging and Performance Degradation of Supercapacitors Integrated into a Modular Multilevel Converter
}

Florian Errigo, Laurent Chédot, Florent Morel, P. Venet, Ali Sari, Alaa Hijazi, Robert Alfie S. Pena

\section{To cite this version:}

Florian Errigo, Laurent Chédot, Florent Morel, P. Venet, Ali Sari, et al.. Assessment of Aging and Performance Degradation of Supercapacitors Integrated into a Modular Multilevel Converter. EPE'20 ECCE Europe, Sep 2020, Lyon, France. pp.1-10, 10.23919/EPE20ECCEEurope43536.2020.9215894 . hal-02987233

\section{HAL Id: hal-02987233 https://hal.science/hal-02987233}

Submitted on 3 Nov 2020

HAL is a multi-disciplinary open access archive for the deposit and dissemination of scientific research documents, whether they are published or not. The documents may come from teaching and research institutions in France or abroad, or from public or private research centers.
L'archive ouverte pluridisciplinaire HAL, est destinée au dépôt et à la diffusion de documents scientifiques de niveau recherche, publiés ou non, émanant des établissements d'enseignement et de recherche français ou étrangers, des laboratoires publics ou privés. 


\title{
Assessment of Aging and Performance Degradation of Supercapacitors Integrated into a Modular Multilevel Converter
}

\author{
F. Errigo ${ }^{1,2}$, L. Chédot ${ }^{1}$, \\ F. Morel ${ }^{1}$ \\ ${ }^{1}$ SuperGrid Institute \\ 23 Rue de Cyprian \\ 69611 Villeurbanne, France \\ florian.errigo@supergrid-institute.com \\ https://www.supergrid-institute.com
}

\author{
P. Venet ${ }^{1,2}$, A. Sari ${ }^{1,2}$, \\ A. Hijazi ${ }^{1,2}$, R. A. Peña ${ }^{1,2}$ \\ ${ }^{2}$ Univ. Lyon, Université Claude Bernard \\ Lyon 1, INSA Lyon, Ecole Centrale de Lyon, \\ CNRS, Ampère, \\ F-69622, Villeurbanne, France \\ http://www.ampere-lab.fr
}

\section{Acknowledgements}

This work was supported by a grant overseen by the French National Research Agency (ANR) as part of the "Investissements d'Avenir" Program ANE-ITE-002-01.

\section{Keywords}

«HVDC», «Multilevel Converter», «Energy storage», «Supercapacitor»

\begin{abstract}
The interest for modular multilevel converter (MMC) with energy storage systems (ESSs) has increased quickly over the last decades. Since an MMC has several hundreds of sub-modules (SMs) and ESS performances usually deteriorate quickly, the ability of the converter to provide the expected service during its lifetime must be investigated. In this work, supercapacitors are used as ESS and the reduction of their capacity is first calculated through lifetime simulation of the ESS using aging models. Then, simulations are used to analyze the available energy of the proposed converter considering dispersions for the initial parameters of ESSs and for the aging rate. Finally, the results are compared with different levels of redundancy and maintenance intervals to discuss the viability of the solution. For the depicted case study, results show that oversizing the ESSs without carrying any maintenance operations during the lifetime of the converter minimizes the total number of cells required.
\end{abstract}

\section{Introduction}

In the upcoming decades, most of the fossil-fuel power plants are planned to be replaced by renewable energy sources connected to the grid through power electronics. This development is particularly critical for the stability of power systems because the transmission grids were not developed to integrate resources with uncontrolled production, located far from the center of consumptions. One effect is the expected decrease of system inertia, which traditionally depends on the kinetic energy stored in the synchronous rotating machines. This implies that can result in dangerously low system frequencies which could lead further to a blackout [1]. This transition must inevitably be accompanied by strengthening the electrical grid.

Grid-scale Energy Storage Systems (ESSs) can be a promising solution to provide fast operating reserves. Because the required overall energy for these new short ancillary services is low compared to traditional bulk storage applications, storage technologies as supercapacitors have recently gained interests to meet these specifications [2]. Usually, utility-scale ESSs are considered to be connected to the grid through dedicated converters. Recent works have studied the opportunity to integrate ESS within converter used for power transfer capabilities such as the Modular Multilevel Converter (MMC) for HVDC (High-Voltage Direct Current) transmission in order to provide ancillary services and, in this way, to extend the range of services they can offer [3]-[5]. 
Even if the energy storage units can be partially distributed among Sub-Modules (SMs) [6], in this work, each SM of an MMC arm has its capacitor connected to a string of supercapacitors by means of a DCDC converter to make an "energy storage sub-module" (ES-SM) (cf. Figure 1). Energy storage technologies are usually based on low-voltage elements while the voltage of a SM capacitor is of the order of one or few thousands of volts. Consequently, the number of components must be inherently high to sustain voltage as well as fulfill the minimum energy requirement.

In addition, the life expectancy of HVDC converters is in the range of decades. As it is often stated a 40-year converter operation is usually expected [7]. This time span is large with regard to the lifetime of electrochemical energy storage technologies. Thus, it is highly challenging for them to maintain high levels of availability throughout this period. In addition, an MMC must remain fully operational between two scheduled maintenance operations to avoid important penalties. Because a 1-GW MMC can have approximately 2400 SMs [8], with a supercapacitor stack, the availability over time of such a solution is a key performance indicator to be considered. Furthermore, the Failure In Time (FIT), 1 FIT equaling 1 failure per billion hours, of the main components in a SM (IGBTs, capacitors, etc.) varies from hundred to few hundreds [7]. Thus, it can be assumed that their failure probability is low during the lifetime of a supercapacitor cell and they are not considered in the following study.

Most of the time, a capacitance decrease or an increase of the equivalent series resistance (ESR) [9], caused by the influence of temperature and voltage, is the main root of supercapacitors performance degradation leading to noncompliance with the expected service. Wear out with age can be a good representation of the outage of supercapacitors. Moreover, the characteristics of these components show natural dispersions. Such dispersion leads to unbalanced aging and uneven energy distribution among cells that can make the system prematurely unable to provide the expected service.

This paper proposes a straightforward method that has never been done before for the assessment of the ability of an MMC with embedded supercapacitors to provide a given service over time. Thanks to physical models and accurate simulation, the lifetime of the storage unit of an ES-SM under a real mission profile is obtained. The inherent probabilistic properties of classical reliability model are avoided. Afterwards, Monte Carlo simulation is done to cope with the uncertainty of the manufacturing process of supercapacitors and the high number of cells to be considered due to the high modularity of an MMC. At the end, the aim of the proposed method is to evaluate the effectiveness of different maintenance intervals and redundancy schemes over the lifetime expectancy of the converter.

The content of the paper is organized as follows. The first section presents the main design parameters of an MMC with ESS and the case study. Secondly, the method to assess the available energy is described. Finally, the last section compares the obtained results, such as the number of supercapacitors required according to different maintenance policies during the lifetime of an HVDC project, and draws the conclusion of this work.

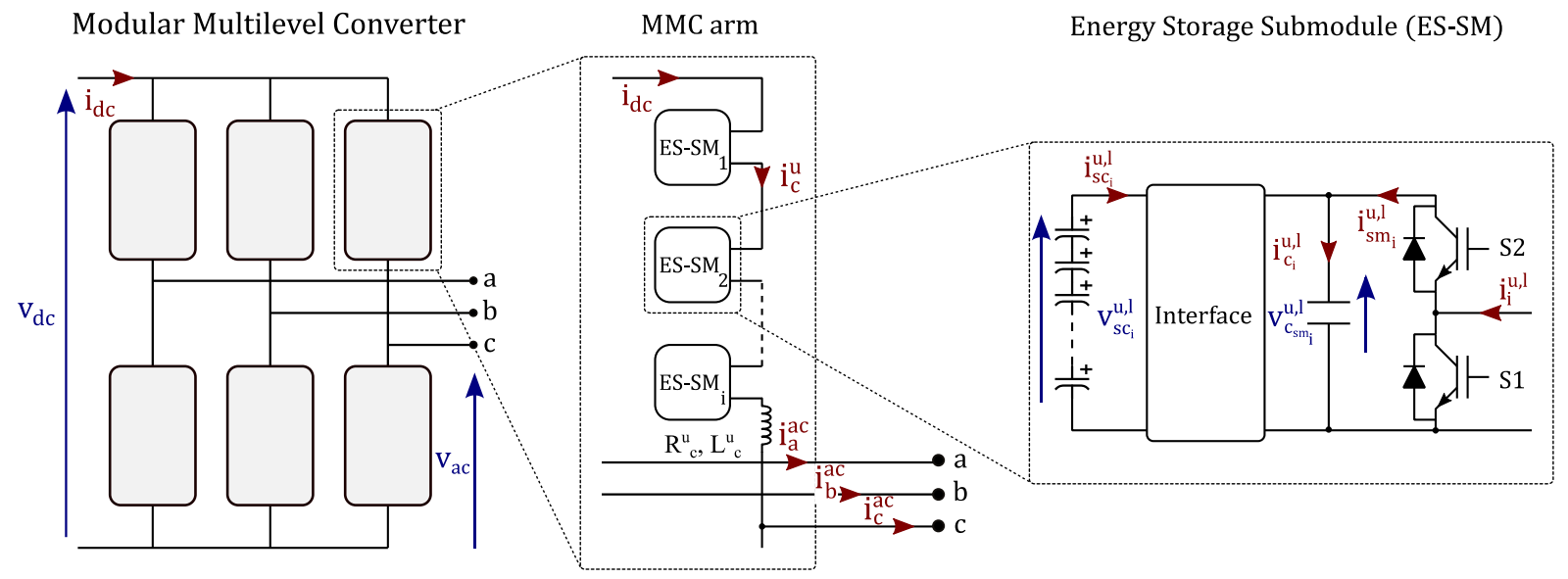

Figure 1 Topology of a modular multilevel converter with integrated energy storage systems 


\section{Modular multilevel converter with energy storage system for fast frequency response}

The purpose of this part is to present the case study and the parameters for the multi-physical modelling and simulation related to the aging of the ESS. Facing future challenges of lacking inertia have introduced the need for short-term dynamic ancillary services from hundreds of milliseconds to tens of seconds, such as fast frequency support. It has been shown that ESS integrated into MMCs are well suited to bring this contribution since they can act quickly and since these service provisions require only a small part of the bulk power flow of a conventional unit, usually less than $50 \mathrm{MW}$ [10].

Recently, some Transmission System Operators (TSO) have launched their own market or products because they required these new fast services from a grid point of view [11]-[14]. By definition, they are used for the purpose to mitigate uncommon system frequency fluctuation in case of disturbance and one can ask of the interest to estimate the availability of such system which are rarely used under those circumstances. However, some specific products deal with the purpose to dynamically manage the normal second-by-second frequency within a tolerance band such as in the United Kingdom (UK) where the National Grid Electricity Transmission (NGET) recently introduced the "enhanced frequency response" (EFR) [11] or in the Nordic system with the standard Normal Frequency Containment Reserves (FCR-N) [15]. The meaning of these services is to provide a one-second real-time proportional response to deviations in the grid frequency to maintain the frequency in the normal range or to provide additional power during a short time until primary reserves reach their maximal set-point or secondary reserves start in case of severe disturbances.

According to the call for tender and public grid frequency data in the UK [11] [16], a one-year load profile mission for the ESS is developed. It is assumed, that the ESS has a maximum power of $50 \mathrm{MW}$ with a storage capacity of $900 \mathrm{MWh}$. It has to provide a proportional response, in either direction, to a change in system frequency with a maximum support duration of 30 seconds when a range, set at $50 \mathrm{~Hz}$ plus or minus $0.05 \mathrm{~Hz}$, is exceed. The output of the ESS can be varied from $\pm 9 \%$ within the frequency deadband to manage its State of Charge (SoC). Figure 2 shows these key features.

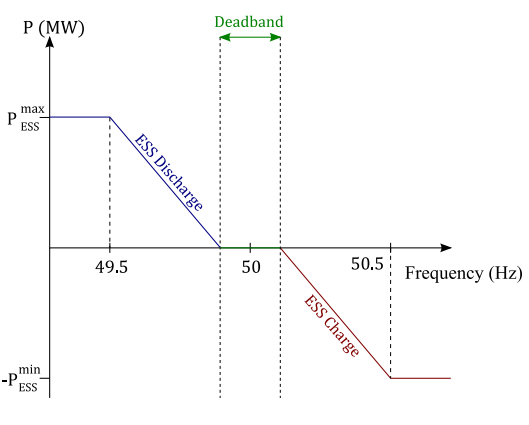

(a)

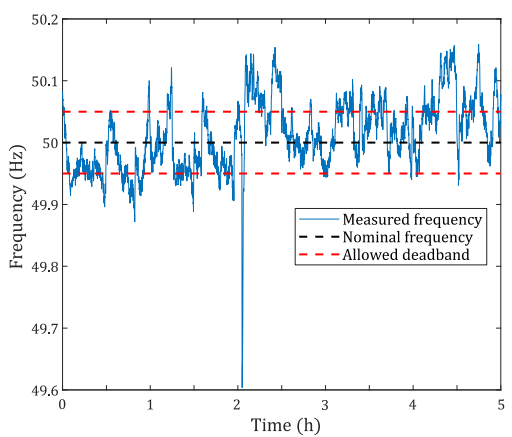

(b)

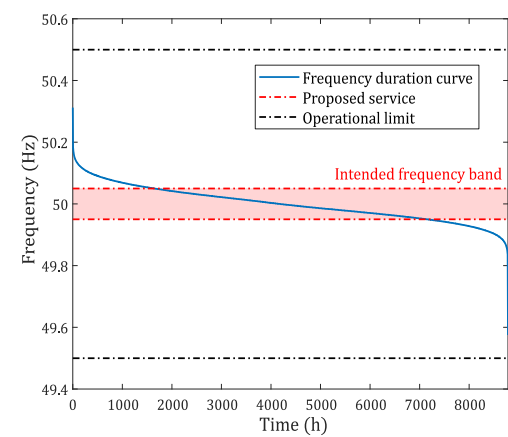

(c)

Figure 2 (a) ESS power response to grid frequency deviation (b) 5 hours extract from the one year second-by-second frequency load profile from the UK system (c) Frequency duration curve of the associated mission profile

Then, a simulation of an MMC ES-SM with a storage function coupled with a multi-physical modelling of the supercapacitor stack has been implemented under MATLAB/Simulink [17][18]. It is carried out throughout the lifetime of the ESS with the previous load profile, which is repeated for every year. The current that will flow through the ESS can be deduced from the simulation to feed the aging model and track ESS lifetime. The obtained data is used to assess the performance of the proposed solution. The main parameters of an ESS per ES-SM and the converter specifications are given in Table I. Note hereinafter in this paper, an energy of 1 p.u. is the energy available in an arm of an MMC for the expected service. 460 cells per SM are needed to provide this energy at the beginning of the lifetime of the station. Due to aging, this level of energy cannot be sustained during all the operating time of the converter and a goal of this work is to determine the number of cells, $N_{\text {cell }}$, to always ensure this energy content. 
Table I: Grid parameters and MMC specifications for the simulation

\begin{tabular}{|l|l|}
\hline Power rating, $P_{m m c}$ & $1 \mathrm{GW}$ \\
\hline DC bus voltage, $v_{d c}$ & $640 \mathrm{kV}$ \\
\hline AC line to ground voltage, $v_{a c}$ & $192 \mathrm{kV}$ \\
\hline Number of ES-SM per arm, $N$ & 400 \\
\hline Sub-module nominal voltage, $v_{c_{s m}}$ & $1600 \mathrm{~V}$ \\
\hline Energy needed per ES-SM for the expected service, $E_{\text {ess }}$ & $375 \mathrm{~kJ}$ \\
\hline Maximum ESS power per ES-SM, $P_{e s s}$ & $20.83 \mathrm{~kW}$ \\
\hline Minimum number of supercapacitors, $N_{\text {cell }}$, per ES-SM & 460 \\
\hline Supercapacitor cell reference & Maxwell Standard Series BCAP0310 \\
\hline Cell capacitance, $C$ & $310 \mathrm{~F}$ \\
\hline Cell Equivalent Series Resistance, $E S R$ & $2.2 \mathrm{~m} \Omega$ \\
\hline Ambient temperature & $55^{\circ} \mathrm{C}$ \\
\hline
\end{tabular}

\section{Energy storage remaining lifetime}

\section{Proposed method}

In reliability studies, the bathtub curve (cf. Figure 3) is widely used to estimate failure rate and thus the lack of ability of an equipment to perform its function [19]. By neglecting, early failures, due to manufacturing process, the main root of failures can be divided into two parts. Random failures which occurs throughout the useful lifetime of a product, with approximately a constant failure rate $\lambda_{0}$, and wear out failures that increase with age.

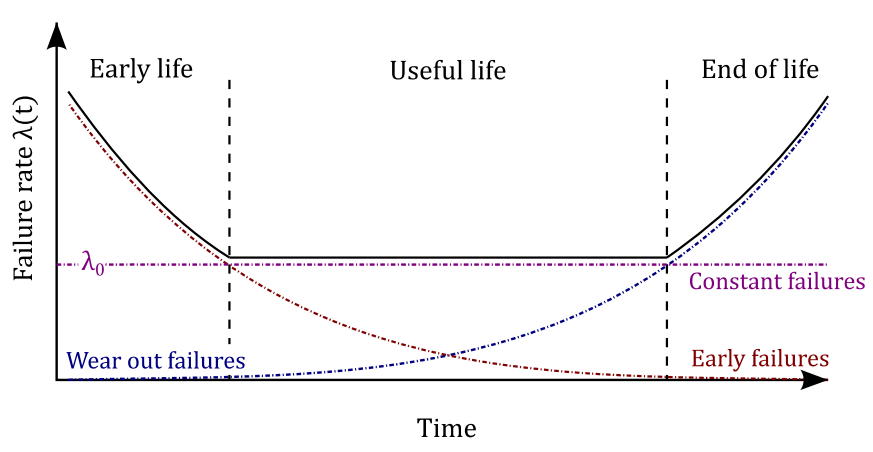

Figure 3 Bathtub curve

However, supercapacitors are electronic components whose FIT is negligible when the failure rate is constant (equal to $\lambda_{0}$ ). Only wear out "failures" can be considered. In fact, applied to supercapacitors, the main failure criteria that are generally considered are a $20 \%$ capacitance loss or a $100 \%$ increase of its equivalent series resistance [9]. It can be clearly seen that these criteria influence primarily the usable energy and does not prevent the ESS to keep working but in a degraded manner. In that case, it does not conduct to an instantaneous loss of ability of the component. Meanwhile, reliability predictions aim at calculating the rate at which a component will fail, based on an estimation of $\lambda_{0}$ from reliability standard, using probabilistic models for failure rate such as exponential or Weibull laws. One of the goals of this paper is to provide an estimation of the degradation of supercapacitor stack's performances integrated within an ES-SM of an MMC through damage analyses. Therefore, aging speed is first studied through electro-thermal simulation to determine ESS lifetime and then statistical analysis are carried out.

\section{System modelling}

In this purpose, the proposed method models the real behavior of components, by applying a specific mission profile and thus the real constraints, to observe the evolution of health indicators such as capacitance. Similarly, in this paper, the assumption that all the supercapacitors within a stack of one ES-SM has the same characteristics is made. Thus, they can be modelled as one equivalent capacitance. Eyring's law can be used to evaluate supercapacitor lifetime [17]. It is considered the main End of Life (EoL) criteria corresponds to a doubling of the initial equivalent series resistance or if the capacitance evolves until $80 \%$ of its initial value. However, this model is valid when a supercapacitor undergoes the same constraints all along its lifetime. This is the reason why, in this work, the modelling of a supercapacitor stack of one ES-SM is based on a dynamic lifetime model from [17]. This latter takes into account that constraints are variable over time and lead to a non-constant degradation rate of the component and so the estimation of the lifetime can change. It considers not only voltage and 
temperature as the main parameters of influence but also current. The dynamic lifetime of a device $\tau_{d}$ is estimated according to the cell voltage, its temperature and the RMS current as shown in equation (1):

$$
\tau_{d}(t)=\frac{\tau_{o}}{\frac{1}{T} \int_{t-T}^{t} \exp \left(\frac{v(t)}{v_{o}}+\frac{\theta(t)}{\theta_{o}}+\frac{i_{r m s}}{i_{r m s_{o}}}\right) d t}
$$

where $v(t)$ and $\theta(t)$ are respectively the instantaneous value of the cell voltage $(\mathrm{V})$ and temperature $\left({ }^{\circ} \mathrm{C}\right)$ while $i_{r m s}$ is the value of the RMS current (A) during the time window considered. $\tau_{o}$ is the theoretical lifetime for a cell at $0 \mathrm{~V}$ and $0{ }^{\circ} \mathrm{C}$ while $v_{0}, \theta_{0}$ and $i_{r m s_{0}}$ are constant whose any decrease of $10{ }^{\circ} \mathrm{C}, 0.2 \mathrm{~V}$ or $30 \mathrm{~A}$ double the lifetime of the component. These parameters are given as follows [17]:

$$
\tau_{0}=439500 \text { years } \quad \theta_{0}=\frac{10}{\ln (2)} \quad v_{0}=\frac{0.2}{\ln (2)} \quad i_{r m s_{0}}=\frac{30}{\ln (2)}
$$

During the simulation, the electrical parameters are provided by the electrical model of the ESS whereas the thermal effects are supplied through a thermal model that analyzes heat transfers and thermal behavior of a supercapacitor cell with the ambient temperature [18]. As reminder, in the literature, the lifetime of a supercapacitor is defined as the time required for a $20 \%$ drop of the capacitance. It does not imply a failure of the component. In this work, this is only used to obtain a model of the evolution of the capacitance over time and we do not refrain from using them beyond this duration.

\section{Simulation results}

In Figure 4, the obtained results are presented. It shows the evolution of the dynamic lifetime $\tau_{d}$ over time. At the beginning of the simulation ( $\mathrm{t}=0$ ), when the ESS experiments a calendar aging, without current constraints, the lifetime is evaluated at 8.45 years. Then, it is clearly observed that estimation is almost constant, with only a slight decrease, when the mission profile is applied. This implies that the degradation rate of the component is not affected by the variation of the thermal and electrical parameters. Under those circumstances, supercapacitors undergo a calendar aging. Consequently, $\tau_{d}$ can be assimilated as a static lifetime and thus the time at the equivalent capacitance of the supercapacitor stack of one ES-SM decreases by $20 \%$, or its ESR doubled, can be determined. It is when the dynamic lifetime estimation equals the simulation time ( $\sim 8.36$ years) as described in Figure 4 .

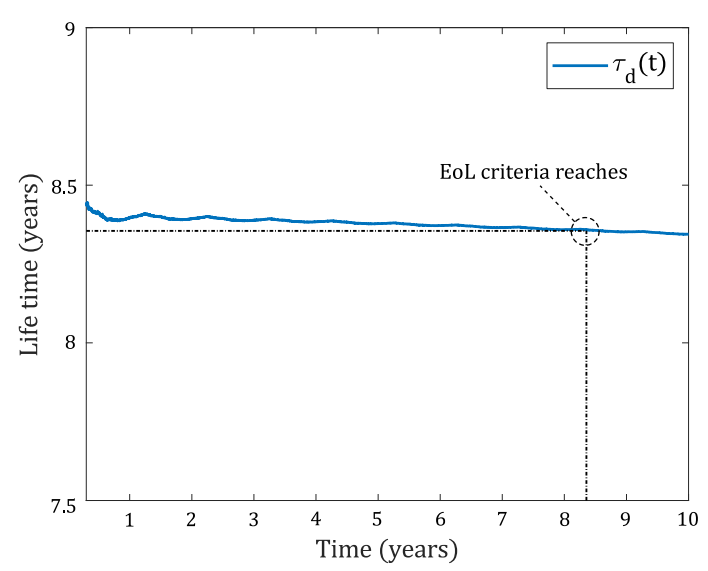

Figure 4 Evolution of the dynamic lifetime $\tau_{d}$

In addition, during the simulation, the temperature of the energy storage device remains the same regardless the variation in power. This is because the grid frequency excursions are most of the time within the intended deadband during one year as seen in Figure 2.(c). It means that the power that has to be supplied is relatively low (cf. Figure 2.(a)) and as a consequence the current that flows through the ES-SM is small with regard to the nominal current that the supercapacitor cell can supply (or absorb) at full power without increasing the device temperature beyond a given value, in that case $40^{\circ} \mathrm{C}$ [9].

This hypothesis is confirmed during the simulation as shown in Figure 5.(b) where the current is always under the nominal current (1 p.u.). In the same manner, the voltage of the ES-SM is always kept close to the voltage reference. This voltage is calculated to make use of $75 \%$ of the maximal stored energy and handle the same amount of available energy for charging as discharging as seen in Figure 5.(a). Throughout this paper, the energy ratio is defined as the available energy from an ES-SM for the expected service and 1 p.u. is the energy content for an arm according to the initial sizing (cf. Table I). 
Based on the previous results, it can be assumed in that situation an ES-SM undergoes calendar aging. Thus, the evolution of the capacitance of an ES-SM over time can be supposed to follow a square root dependency law characteristic of a floating aging that traduced the formation of a solid layer at the interface electrode/electrolyte [20], as shown in equation (5) with $A_{c}$ a proportional coefficient.

$$
C_{s c}(t)=C_{s c 0}-A_{c} \sqrt{t}
$$

Finally, according to the knowledge of the time at which the capacitance drops by $20 \%$ thanks to the simulation, it is easy to extrapolate the value of this coefficient with equation (5).

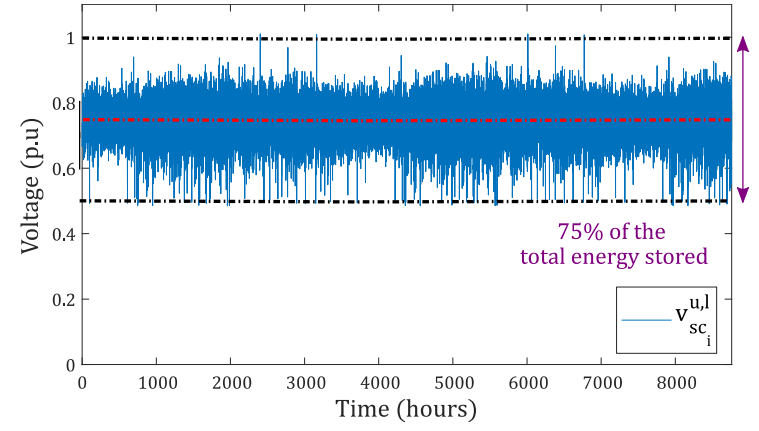

(a)

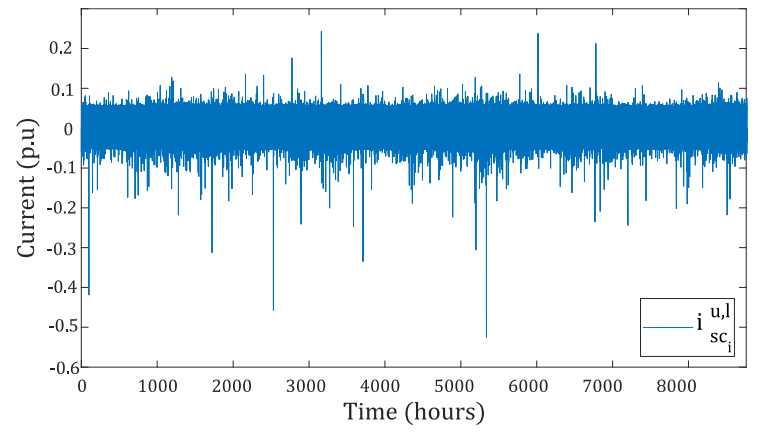

(b)

Figure 5 Simulation results for the 1st year with: (a) Voltage at the terminal of the supercapacitor stack of one ES-SM (b) Current that flows through it

\section{Monte Carlo simulation for reliability evaluation}

\section{Probabilistic method}

Generally, reliability modelling uses statistical techniques based on reliability standards such as US Military Handbook $217 \mathrm{~F}$. Thus, probabilistic analysis is made assuming a constant failure rate or by introducing a time varying failure rate to define the expected lifetime of a device [7,21]. These techniques are often criticized because of the differences between field results and predictions [19]. Moreover, these mathematical models can quickly become complex when it models periodic maintenance and the provision of redundancy, where SMs are at different levels of aging. In this paper, the wear out of components under a load profile is used to calculate performance degradation before carrying out well-known Monte Carlo methods to an MMC.

The above-mentioned simulations focus only on one ES-SM. Therefore, it is necessary to evolve from component level to converter level since an MMC contains six arms comprising hundreds of ES-SMs. as shown in Figure 1. It is assumed that all the ES-SMs are assembled identically to describe an arm with redundancy, which means they all share load during normal operation. It implies an active redundant mode. Similarly, an ESS in a SM is just a series connection of supercapacitors. Otherwise, the total stored energy of an ES-SM can be easily estimated by observing the degradation rate of a stack and subsequently the available energy in an arm to assess its lack of ability to complete its intended function. Because in average, the six arms of an MMC behave identically, it is possible to restrict the study to only one converter arm.

Note that supercapacitors have natural dispersion in capacitance or ESR due to their manufacturing but also in reason of aging. Similarly, the kinetic of degradation of the capacity also expect discrepancies. In addition, a stack of supercapacitors of one ES-SM can be replaced during maintenance operations. As a consequence performance degradation of ES-SMs within an MMC is not uniform over time because they experience different levels of aging. To define the availability of a storage function within an $\mathrm{MMC}$ according to these dispersions, a sequential Monte Carlo simulation has been implemented. This flexible approach allows to consider the time progress and take into account repairable systems. By random sampling of ES-SM characteristics with a given probability distribution, a high number of independent lifetime scenarios can be generated and played. From these simulations, performance indices are measured and compared. The method is as follows. 
A normal probability distribution is used to randomly select the initial capacitance assuming $95 \%$ of production samples have a value around $\pm 20 \%$ the typical capacitance value of an ES-SM as shown in Figure 6.(a). The minimum number of supercapacitors, $N_{\text {cell }}$, per ES-SM to provide the expected service is defined at the initialization stage. As shown, in Table I, one row of 460 cells of $310 \mathrm{~F}$ is necessary. It leads to an equivalent supercapacitors of $0.67 \mathrm{~F}$. The same process is applied to the degradation speed of the capacitance (ie. slope deviation) around the average value of the coefficient $A_{c}$ of (5), found with the simulation described in the previous paragraph, at the beginning of each scenario (cf. Figure 6.(b)).

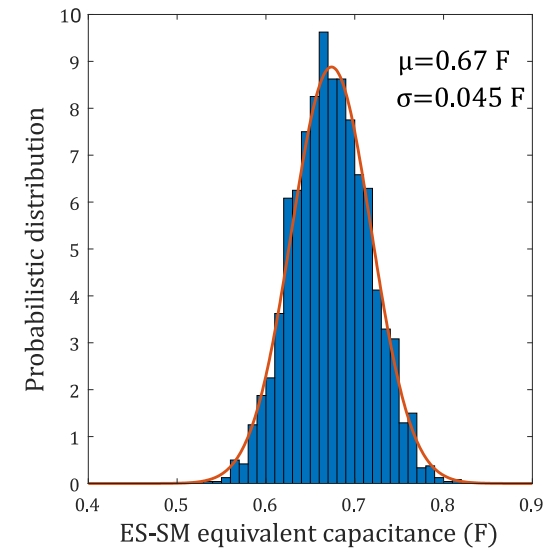

(a)

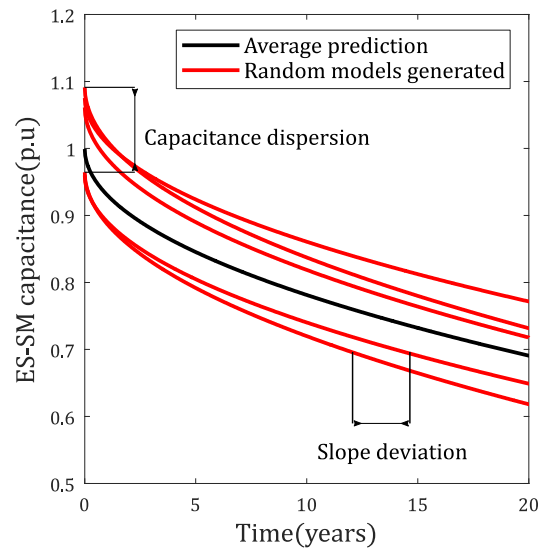

(b)

Figure 6 (a) ES-SM capacitance distribution (b) Example of a random selection of five ES-SMs with slope and initial capacitance dispersion

Then, the capacity of all ES-SMs is observed during all the life expectancy of the converter. So, the evolution of the total stored energy from the storage function can be estimated over time and thus the energy available for the service. Afterward, the availability function of the ESS of the MMC can be drawn depending on the desired energy threshold. As a reminder, in this study, the availability corresponds to the ability to provide a minimal level of energy in case of disturbance to sustain the grid. However, because aging was not considered during the design phase, it is expected that the energy requirement will not be ensured due to performance degradation. To make sure that the available energy remains above the desired threshold, redundant cells are placed in series within an ES-SM. Thus, the scenario is restarted as long as the minimum number of cells to be inserted, allowing the desired availability of the storage function, is not found. It is considered that increasing the number of cells in series allows to increase the total stored energy per stack by a factor $N_{\text {cell }}$ if they are all charged at its nominal voltage. Note that the topology of the interface converter and the cell configurations are not looked at for these redundant designs.

Scheduled events, such as different preventive maintenance policies, are also included in the model. As an example, at each maintenance interval $T_{m}$ (years), all the stacks of supercapacitors of the ES-SMs that reach a threshold capacitance value $C_{\min }$, which corresponds to a certain decrease in the rated capacitance, are replaced by a new one while the others are still operational but with a lower capacity than when they were commissioned. Note that if an ES-SM overcomes the threshold capacitance before a maintenance interval, it is still operational even if its energy content is highly reduced.

The combination of which will provide an indication of the effects from oversizing the energy storage systems within a SM and varying the maintenance interval to maintain proper operation of the energy storage function throughout all the HVDC project lifetime. To conclude, the Monte Carlo estimation is reproduced many times for each scenario and the results analyzed for the average availability of the case study.

\section{Simulation results}

The results generated from the Monte Carlo simulations are presented in this part. The estimation was reproduced 100 times for each case. Firstly, the influence of oversizing the ES-SMs to sustain different 
energy levels at the end of the lifetime of the converter without any maintenance operations is described. Hence, Figure 7.(a) depicts the evolution of the energy available in one arm from the ESSs over time while Table II shows that as the energy threshold increases, the number of cells per ES-SM.

Table II: Minimum number of cells per ES-SMs to ensure different energy requirements for the expected service at the end of the lifetime of the converter without maintenance

\begin{tabular}{|l|c|c|c|c|}
\hline & \multicolumn{4}{|c|}{ Energy requirements at 40 years } \\
\hline Energy (p.u.) & Initial sizing & 0.8 & 0.9 & 1 \\
\hline Number of cells $N_{\text {cells }}$ & 460 & 655 & 736 & 818 \\
\hline
\end{tabular}

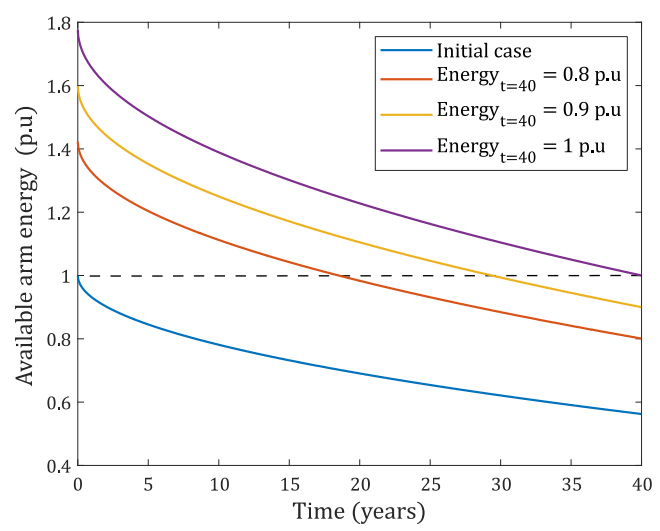

(a)

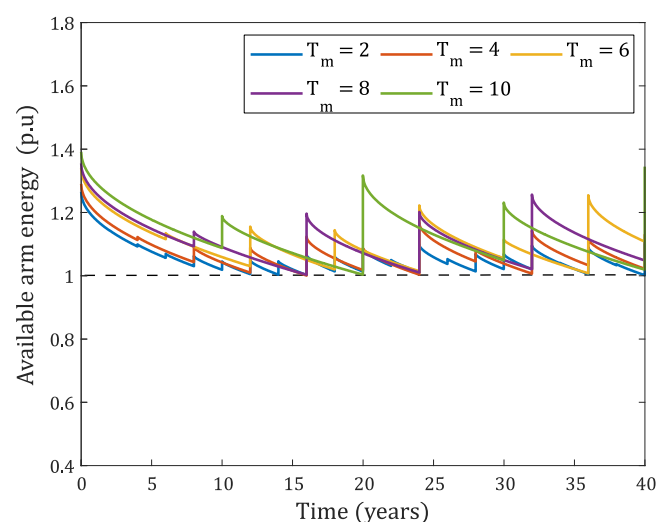

(b)

Figure 7 (a) Available arm energy for the expected service for different energy requirements at the end of life of the converter without maintenance (b) Available arm energy for the expected service for different maintenance intervals with a full availability

Obviously, the greater the initial energy available, the more redundant cells needed. It can be observed the initial sizing, that allows 1 p.u. of available energy at the commissioning, leads to less than 0.6 p.u. at the end of the lifetime of the converter. Otherwise, 818 cells per ES-SM are needed to ensure an energy level greater than 1 p.u., which implies to oversize the ESS by $80 \%$. Finally, it should be noted in that case, each increment of 0.1 p.u. at 40 years requires a constant number of extra cells. However, this solution can face some difficulties. In fact, it is assumed supercapacitors are still used even if what is called "EoL criteria" in the literature is reached, which stands for a $20 \%$ drop in their initial capacitance. As observed in Figure 7.(a), most of the cells would lose approximately $45 \%$ of their rated value during the time span of the project which is much more than what is considered in most aging studies in the literature. Therefore, it should be clarified experimentally whether or not supercapacitors can be used with such a low capacity without leading to important concerns from a reliability point of view. In fact, the aging of supercapacitors is accompanied by a loss of performance but also with gases development. The main risk is an overpressure inside the cell that can guide to a cracking of the component or in the worst case to a blast.

In a second step, the same process is reproduced considering a maintenance interval $T_{m}$. This latter is varied from 2 to 10 years with a step time of 2 years. Indeed, an HVDC converter station scheduled outage is regularly planned for maintenance activities. All the ES-SMs, for which the capacity fade under the threshold capacitance value $C_{\min }$ at each maintenance operation, are replaced. In Figure 7.(b), that shows the evolution of the energy available from the energy storage units over time, it was decided to replace it when they reached a milestone of $70 \%$ of the initial capacitance. Similarly, an arm must keep a target availability for the expected service of 1 p.u. during the lifetime of the converter, whatever the maintenance interval, that correspond to the minimum energy needed as explained previously. Figure 7.(b) does not follows a similar shape as in Figure 7.(a) because an increase of energy is brought at each maintenance intervals. Thus, the availability is improved. Furthermore, the number of cells per ES-SM to keep an energy level for the expected service greater than 1 p.u. is reduced compared to the same case without maintenance activities. 
Clearly in that case, the available energy is a combination of the total number of supercapacitors per ESSMs and the number of time this stack has to be changed, which implies an optimum configuration. For this purpose, the relationship between the number of supercapacitors in a stack per ES-SM and the capacitance for which they are changed according to different maintenance intervals is presented in Figure 8.(a). Figure 8.(b) illustrates in the same manner the number of stacks that should be changed while Figure 8.(c) describes the total thousands of supercapacitors required during all the lifecycle of the converter.

Less frequent maintenance needs more cells per ES-SM but less stacks of supercapacitors to be replaced. This latter is explained by the fact that each stack in all ES-SMs are oversized with regard to the capacitance threshold. Similarly, decreasing the capacitance threshold allows less replacement. These choices lead to an important gain with less supercapacitors needed during the lifetime of the converter (cf. Figure 8.(c)), even if it is clearly necessary to oversize the system (cf. Figure 8.(a)). At the same time, a regular change of the stack of an ES-SM is not necessarily the best choice. Moreover, such maintenance activities are usually expensive particularly for offshore converters. In order to highlight this assessment and compare the different strategies, the total number of cells required to have at least 1 p.u. of available energy all the time, and so a full availability, with any maintenance is plotted in dashed line on Figure 8.(c). Undoubtedly, this strategy to regularly have maintenance suffers of the quick decrease of capacitance at the beginning of the lifetime of a supercapacitor, due to the square root dependency, that leads to often change cells. As shown in Figure 7.(a) with no maintenance operations, a $20 \%$ decrease in capacity is expected during the first 8.4 years but only another loss of $25 \%$ is noticed during the last 31.6 years. There is an obvious trade-off that concerns the capacitance threshold for which supercapacitors have to be changed against the risk of failures due to significant aging. Nonetheless, it seems that oversizing the energy storage system without carrying any maintenance operations or the bare minimum seems a pertinent solution.

(a)

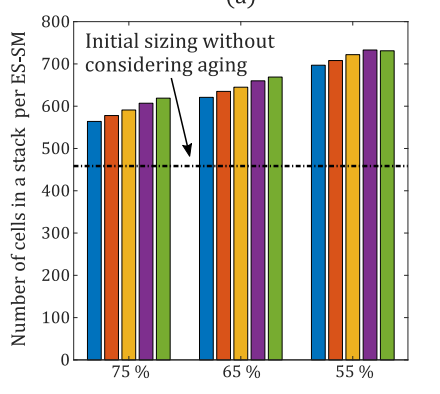

(b)

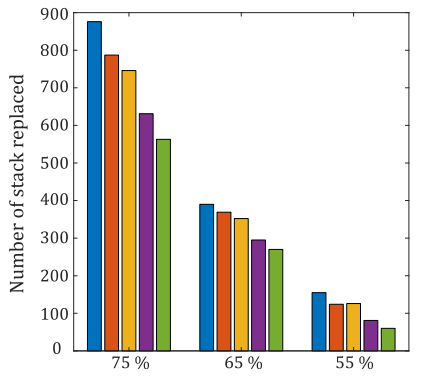

(c)

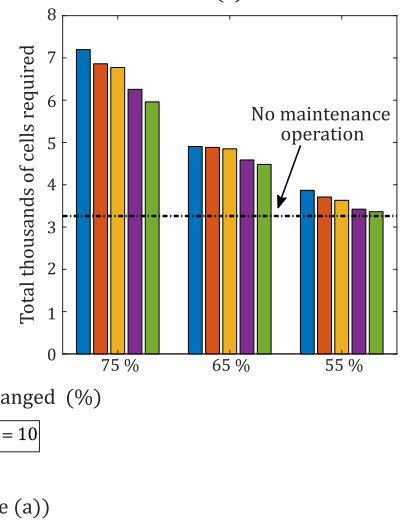

Figure 8 (a) Number of supercapacitors in a stack per ES-SM against capacitance threshold according to different maintenance intervals (b) Number of stacks of supercapacitors replaced against capacitance threshold according to different maintenance intervals (c) Total thousands number of cells capacitance threshold according to different maintenance intervals

\section{Conclusion}

This paper proposes a general methodology with a practical application that considers the physics of aging mechanisms to analyze performance degradation of ESSs integrated into an MMC during the lifetime of an HVDC project. A multi-physical simulation was conducted to estimate the expected lifetime of one supercapacitor stack under a high resolution mission profile. Then, a lifetime model of a supercapacitor stack to track the evolution of the capacitance as a function of time was defined. Afterwards, Monte Carlo simulations were performed to evaluate the availability of a storage service provided by an MMC under different redundancy and maintenance interval schemes.

Results show that increasing the number of planned maintenance lead to an important increase of the number of supercapacitor stacks required during the project. It was also found that oversizing the ESS at the beginning of the lifetime of the converter per ES-SM allows to minimize maintenance activities, 
which are expensive tasks, and to keep a good level of energy to fulfill requirements. However, it is done at a cost of using a supercapacitor beyond its normal utilization range. It will be necessary to evaluate if it does not lead to a high probability of failure. In future works also, cost assessment indicators should be equally proposed to evaluate the cost effectiveness of both strategies to define an optimum with regard to the total cost associated. Finally, the proposed methodology can potentially be extended to other energy storage technologies such as batteries.

\section{References}

[1] P. Tielens, D. Van Hertem, "The relevance of inertia in power systems", Renewable and Sustainable Energy Reviews, 2016, vol.55, pp. 999-1009.

[2] R. Alvarez, M. Pieschel, H. Gambach, and E. Spahic, "Modular multilevel converter with short-time power intensive electrical energy storage capability," in 2015 IEEE Electrical Power and Energy Conference (EPEC), Oct 2015, pp. 131-137

[3] P. Judge and T. Green, "Modular multilevel converter with partially rated energy storage with intended applications in frequency support and ancillary service provision," IEEE Transactions on Power Delivery, pp. 1$1,2018$.

[4] I. Trintis, S. Munk-Nielsen, and R. Teodorescu, "A new modular multilevel converter with integrated energy storage," in IECON 2011-37th Annual Conference on IEEE Industrial Electronics Society. IEEE, 2011, pp. 10751080 .

[5] W. Zeng, R. Li, and X. Cai, “A New Hybrid Modular Multilevel Converter with Integrated Energy Storage," IEEE Access, pp. 1-1, 2019.

[6] F. Errigo, L. Chédot, P. Venet, A. Sari, P. Dworakowski and F. Morel, "Assessment of the Impact of Split Storage within Modular Multilevel Converter," IECON 2019 - 45th Annual Conference of the IEEE Industrial Electronics Society, Lisbon, Portugal, 2019, pp. 4785-4792.

[7] J. Wylie, M. C. Merlin, and T. C. Green, "Analysis of the effects from constant random and wear-out failures of sub-modules within a modular multi-level converter with varying maintenance periods," in 2017 19th European Conference on Power Electronics and Applications (EPE'17 ECCE Europe, 2017)

[8] J. Peralta, H. Saad, S. Dennetière, J. Mahseredjian, and S. Nguefeu, "Detailed and averaged models for a 401 level MMC-HVDC system," IEEE Transactions on Power Delivery, vol. 27, no. 3, pp. 1501-1508, 2012

[9] Maxwell Technologies, Inc., Application Note

[10] C. Mosca et al., "Mitigation of frequency stability issues in low inertia power systems using synchronous compensators and battery energy storage systems," in IET Generation, Transmission \& Distribution, vol. 13, no. 17, pp. 3951-3959, 392019.

[11] National Grid. Enhanced frequency response; 2016.

[12] Australia Energy Market Operator, "Fast frequency response in the NEM,” Tech.Rep., 2017.

[13] ENTSO-E, "Technical Requirements for Fast Frequency Reserves Provision in the Nordic Synchronous Area," Tech. Rep., 2020.

[14] EirGrid, "DS3 System Services Implementation Project - Regulated Arrangements," Tech. Rep., 2019.

[15] ENTSO-E, "Nordic Balancing Philosophy” Tech. Rep., 2016.

[16] National Grid. Available: https://www.nationalgrideso.com/balancing-services/frequency-responseservices/historic-frequency-data

[17] P. Kreczanik, P. Venet, A. Hijazi, and G. Clerc, "Study of Supercapacitor Aging and Lifetime Estimation According to Voltage, Temperature, and RMS Current," IEEE Trans. Ind. Electron., vol. 61, no. 9, pp.4895-4902, Sep. 2014.

[18] A. Hijazi, P. Kreczanik, E. Bideaux, P. Venet, G. Clerc, and M. Di Loreto, "Thermal Network Model of Supercapacitors Stack," IEEE Trans. Ind. Electron., vol. 59, no. 2, pp. 979-987, Feb. 2012

[19] P. Venet, "Amélioration de la sûreté de fonctionnement des dispositifs de stockage d'énergie“, Université Claude Bernard, Lyon, HDR 2007

[20] R. German, A. Sari, P. Venet, Y. Zitouni, O. Briat and J. Vinassa, "Ageing law for supercapacitors floating ageing," 2014 IEEE 23rd International Symposium on Industrial Electronics (ISIE), Istanbul, 2014, pp. 1773-1777.

[21] B. Wang, X. Wang, Z. Bie, P. D. Judge, X. Wang and T. C. Green, "Reliability Model of MMC Considering Periodic Preventive Maintenance," in IEEE Transactions on Power Delivery, vol. 32, no. 3, pp. 1535-1544, June 2017. 